\title{
Publicações de enfermeiros sobre álcool e alcoolismo em anais do Congresso Brasileiro de Enfermagem
}

\author{
Literary production of nurses on alcohol and alcoholism published \\ in the Annals of the Brazilian Nursing Congress \\ Producción literaria de enfermeras sobre alcohol y alcoholismo publicada \\ en los Anales del Congreso Brasileño de Enfermería
}

\section{Divane de Vargas', Janaina Soares'}

'Universidade de São Paulo, Escola de Enfermagem, Departamento de Enfermagem Materno-Infantil e Psiquiátrica, Programa de Pós-Graduação em Enfermagem. São Paulo-SP, Brasil.

\author{
Submissão: 04-03-2011 Aprovação: 28-04-2013
}

\section{RESUMO}

Este é um estudo exploratório, de cunho bibliográfico, cujo objetivo foi verificar e caracterizar a produção literária de enfermeiros sobre a temática álcool e alcoolismo publicada nos anais do Congresso Brasileiro de Enfermagem publicados no período de 1998 a 2008. A amostra constituiu-se de 99 resumos diretamente relacionados ao tema. Os dados foram categorizados segundo os pressupostos da análise de conteúdo e originaram sete categorias para análise. Evidenciou-se que houve um aumento da produção sobre a temática nos últimos cinco anos e que o atendimento ao paciente alcoolista foi o tema mais pesquisado pelos enfermeiros. Concluiu-se que, apesar do aumento da produção sobre o tema, esta ainda é incipiente e que outros estudos dessa natureza merecem ser realizados, de forma a mapear a produção da enfermagem divulgada nos eventos científicos da área.

Descritores: Enfermagem; Alcoolismo; Saúde Mental.

\begin{abstract}
This is an exploratory study, of bibliographical type, which aimed to verify and characterize the literary production of nurses on the topic of alcoholism published in the Annals of the Brazilian Nursing Congress, published in the period of 1998-2008. The sample consisted of 99 abstracts that were directly related to the topic. The data were categorized according to the assumptions of content analysis and resulted in seven categories of analysis. It was evident that there was an increase in the production on the subject over the period of 1998-2008, and that the alcoholic patient care was the theme most researched by nurses. It was concluded that, despite the increase in production on the subject, it is still in its infancy, suggesting that other studies of this nature are needed in order to map the production of nursing published in the scientific events in the area.
\end{abstract}

Key words: Nursing; Alcoholism; Mental Health.

\section{RESUMEN}

Se trata de un estudio exploratorio, de tipo bibliográfico, que tuve por objeto verificar y caracterizar la producción literaria de las enfermeras sobre el tema del alcoholismo, publicada en los Anales del Congreso Brasileño de Enfermería, en el período de 1998-2008. La muestra estuvo conformada por 99 resúmenes que estaban directamente relacionados con el tema. Los datos se clasifican de acuerdo a los supuestos de análisis de contenido y resultaron en siete categorías de análisis. Era evidente que hubo un aumento en la producción sobre el tema en el período de 1998-2008, y que la atención al paciente alcohólico fue el tema más estudiado por los enfermeros. Se concluyó que, a pesar del aumento de la producción sobre el tema, esto todavía está en su infancia, lo que sugiere que se necesitan otros estudios de este tipo con el fin de asignar la producción de la enfermería publicada en Anales de eventos científicos en el área.

Palabras clave: Enfermería; Alcoholismo; Salud Mental.

\section{AUTOR CORRESPONDENTEＪanaina Soares E-mail: janainasoaresjs@hotmail.com}




\section{INTRODUÇÃO}

Atualmente, o consumo de álcool constitui um dos mais significativos riscos para a saúde em todo o mundo. Segundo o Relatório sobre da Saúde Mundial(1), são atribuídos ao álcool 4\% da carga de morbilidade, o equivalente a 58,3 milhões dos anos de vida perdidos, ajustados em função da deficiência/doença (AVAD), e 1,8 milhões de falecimentos. Nas Américas, o álcool é o principal fator de risco entre os 27 fatores avaliados na carga de morbidade ${ }^{(2)}$. No Brasil, o último levantamento domiciliar realizado nas 108 maiores cidades ${ }^{(3)}$ apontou que 12,3\% das pessoas entre 12 e 65 anos são dependentes de álcool e que $74,6 \%$ já consumiram álcool na vida. Os índices encontrados revelam que o uso e o abuso de álcool caracterizam-se como dos maiores fatores de risco e agravos à saúde da população ${ }^{(4)}$, e é consistente o fato de que estejam associados a mais de 60 condições nosológicas relacionadas no CID 10 $10^{(5)}$.

Apesar das consequências na saúde da população e do frequente contato estabelecido entre o enfermeiro e os usuários de álcool e alcoolistas nos serviços de saúde, o tema álcool e outras drogas não tem constituído área de interesse de pesquisa entre estes profissionais ${ }^{(6)}$. No entanto, segundo pesquisa publicada em $2005^{(6)}$, o descuido sobre o tema não constitui exclusividade da enfermagem uma vez que, diante da magnitude da problemática relacionada ao álcool e ao alcoolismo na saúde da população, poucos estudos têm sido realizados sobre esta questão em toda a área da saúde. No que compete à enfermagem, esse mesmo estudo ${ }^{(6)}$ aponta que o fato de a classe pouco produzir sobre a questão tem refletido no pouco impacto na investigação da temática álcool e outras substâncias psicoativas, o que leva as autoras a concluir que não se estão realizando novas investigações e estudos ou que, talvez, não esteja sendo adequadamente divulgada sua produção ${ }^{(6)}$.

Além da escassez de estudos sobre o tema álcool e outras drogas na literatura da enfermagem, são poucos os estudos que se têm ocupado em mapear a produção da área sobre a temática. No único estudo publicado com objetivo de identificar o conhecimento produzido pela enfermagem brasileira na temática álcool e outras drogas - como BIREME - BDEnf (Base de dados da Enfermagem), Acervo de Teses e Dissertações da Universidade de São Paulo e consultas adicionais a três periódicos da área -, as autores utilizaram bases de dados para coleta das informações. Os resultados da pesquisa permitiram observar que é pequena a produção cadastrada nas bases de dados disponíveis online, pois, dos 6.251 resumos selecionados pelos descritores, apenas 3,4\% foram produzidos por enfermeiros e se referiam ou faziam alusão ao tema álcool e outras substâncias psicoativas.

No que se refere aos temas abordados pelos enfermeiros em suas pesquisas sobre o assunto, o estudo ${ }^{(6)}$ evidenciou que se destacavam aqueles relacionados ao tratamento (principalmente do alcoolismo), ao ensino a respeito e a levantamentos realizados em populações vulneráveis, com jovens e crianças. As lacunas apontadas na produção do conhecimento da enfermagem sobre a questão foram as relacionadas a estudos com idosos e à violência, tendo sido constatado que, particularmente quanto a esta última, o predomínio da produção compete a outras áreas da saúde que não a enfermagem.
Partindo-se da constatação de que a literatura de enfermagem sobre o tema álcool e outras drogas é escassa e considerando-se que seja possível que muitas produções não se tenham revertido em publicações, periódicos, ou não constituam resultados de teses ou dissertações e consequentemente não componham as bases formais de dados, torna-se importante levantar informações em outras fontes, como os anais de resumos de eventos científicos sobre a área. Frente a isso, no presente estudo as fontes para obtenção de informações foram os Anais do Congresso Brasileiro de Enfermagem.

A escolha desse evento se deve ao fato de ele ser considerado um dos mais antigos e tradicionais acontecimentos científicos da área de enfermagem e porque, desde seu início, em 1947, ele tem representado possibilidade de diálogo na construção do exercício da profissão, na busca de compreender e transformar a amplitude de atuação, com reflexo sobre a história e a prática dos profissionais de enfermagem. Assim, considera-se que os Congressos Brasileiros de Enfermagem (CBEn), ao refletir sobre a situação atual da enfermagem brasileira, têm se constituído uma instância de construção do conhecimento nesta área que se pode estender para a produção literária do enfermeiro / enfermagem no campo das substâncias psicoativas, especificamente no estudo relacionado a álcool e alcoolismo ${ }^{(7)}$.

\section{OBJETIVO}

Verificar e caracterizar a produção de enfermeiros sobre álcool e alcoolismo, publicada nos Anais do Congresso Brasileiro de Enfermagem (CBEn), no período compreendido entre 1998 e 2008.

\section{MATERIAL E MÉTODO}

Trata-se de um estudo exploratório descritivo, realizado por meio de levantamento bibliográfico nos Anais do Congresso Brasileiro de Enfermagem (CBEn) no período de 1998 a 2008. Foram analisados 11 Anais de resumos - três, disponibilizados na forma impressa (1998 a 2000), e oito, em CD ROOM (2001 a 2008). Para o levantamento dos dados, utilizaram-se os seguintes descritores: 1. Álcool; 2. Alcoolismo e 3. Substâncias psicoativas. Os critérios de inclusão na amostra do estudo foram: constar de qualquer um dos descritores mencionados nas palavras-chaves e versar sobre a temática álcool e alcoolismo. Satisfizeram os critérios de inclusão 99 resumos que estavam diretamente relacionados ao tema proposto. De posse do material, os dados foram organizados segundo ano de publicação, frequência das categorias e subcategorias e procedência geográfica e institucional.

A segunda etapa do estudo consistiu-se de análise qualitativa realizada por meio da categorização dos dados, ou seja, do agrupamento de elementos com características comuns relacionadas entre si. O procedimento de categorização representa o agrupamento de ideias ou expressões a respeito de um conceito comum capaz de abranger todo o conjunto de dados ${ }^{(8)}$. Essa análise originou sete categorias temáticas para apresentação: I. Levantamentos populacionais; II. Assistência de enfermagem; III. Populações vulneráveis; IV. Sentimentos / concepções e atitudes 
de enfermeiros frente ao álcool e ao alcoolismo; V. Ensino sobre Álcool e Outras Drogas (AOD); VI. Concepções / vivências do alcoolista; VII. Alcoolismo e família. Essas categorias foram sintetizadas de modo a apresentar a frequência dos temas pesquisados e publicados pelos enfermeiros nos Anais do CBEn no período de 1998-2008.

\section{RESULTADOS}

\section{1) Análise geral do material}

Observou-se que, dos 99 resumos publicados no período abrangido pela pesquisa, a maior concentração de trabalhos sobre a temática álcool e alcoolismo (15\%) ocorreu no ano de 2006, seguida do percentual de 12\% no ano de 2008 (Gráfico 1).

Com relação aos aspectos relacionados ao álcool e ao alcoolismo apresentados no CBEn e publicados em seus Anais, observou-se que: em sua maioria (32\%), os resumos se
Gráfico 1 - Distribuição dos resumos publicados sobre a temática álcool e alcoolismo nos anais do CBEn conforme o ano de publicação, São Paulo, 2010.

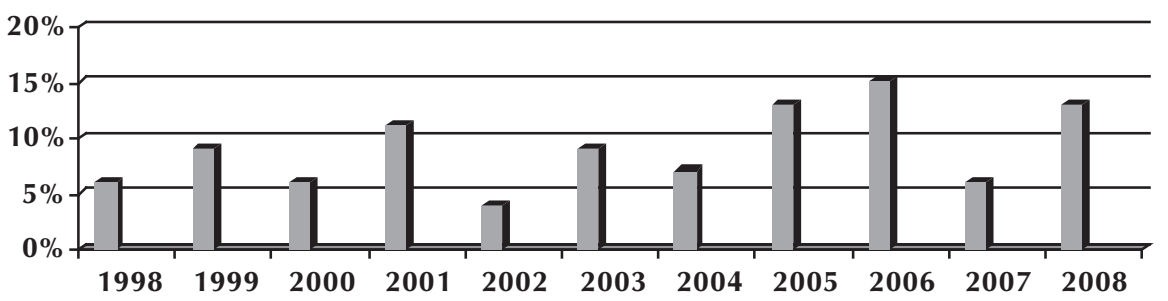

Tabela 1 - Distribuição dos temas dos trabalhos sobre álcool e alcoolismo nos anais CBEn entre 1998 a 2008 de acordo com frequência das categorias e subcategorias identificadas, São Paulo, 2010.

\begin{tabular}{|c|c|c|c|c|c|}
\hline \multirow{2}{*}{ Categorias } & \multicolumn{2}{|c|}{ Produção } & \multirow{2}{*}{ Subcategorias } & \multicolumn{2}{|c|}{ Produção } \\
\hline & $\mathrm{N}$ & $\%$ & & $\mathbf{N}$ & $\%$ \\
\hline \multirow[t]{4}{*}{ I.Levantamentos Populacionais } & 27 & 27 & Serviços de Saúde & 12 & 44 \\
\hline & & & Instituições de Ensino & 7 & 26 \\
\hline & & & Populações Específicas* & 3 & 12 \\
\hline & & & Demandas espontâneas & 5 & 18 \\
\hline \multirow[t]{3}{*}{ II.Assistência de Enfermagem } & 32 & 32 & Assistência ao indivíduo & 26 & 80 \\
\hline & & & Assistência à família & 4 & 12 \\
\hline & & & Assistência ao indivíduo e a família & 3 & 8 \\
\hline \multirow[t]{4}{*}{ III.Populações Vulneráveis } & 27 & 27 & Mulheres & 6 & 22 \\
\hline & & & Adolescentes & 8 & 30 \\
\hline & & & Universitários & 9 & 33 \\
\hline & & & Populações específicas* & 4 & 15 \\
\hline \multirow[t]{3}{*}{ IV.Sentimentos, Concepções e Atitudes de Enfermeiros } & 6 & 6 & Uso/abuso de álcool & 1 & 13 \\
\hline & & & Alcoolismo & 3 & 37 \\
\hline & & & Alcoolista & 4 & 50 \\
\hline \multirow[t]{3}{*}{ V.Ensino sobre Álcool e Outras Drogas } & 3 & 3 & Instrumentos de coleta & 1 & 33 \\
\hline & & & Atitudes de estudantes & 1 & 34 \\
\hline & & & Conteúdos ministrados & 1 & 33 \\
\hline \multirow[t]{3}{*}{ VI.Concepções e Vivências do Alcoolista } & 7 & 7 & Concepções e conhecimentos & 4 & 57 \\
\hline & & & Necessidades do indivíduo & 1 & 14 \\
\hline & & & Fatores Desencadeantes & 2 & 29 \\
\hline \multirow[t]{4}{*}{ VII.Alcoolismo e Família } & 6 & 6 & Relação família / alcoolista & 1 & 17 \\
\hline & & & Repercussões & 2 & 33 \\
\hline & & & Recursos públicos de apoio & 1 & 17 \\
\hline & & & Perfil familiar & 2 & 33 \\
\hline Total & 99 & & & 108 & \\
\hline
\end{tabular}

*Taxistas, motoristas de caminhões e portadores de comorbidades crônicas. 
em mais de uma categoria e, dos 18 resumos que se repetiram em mais de uma categoria, 11 foram reorganizados nas categorias populações vulneráveis e levantamentos e 7, nas categorias populações vulneráveis e assistência de enfermagem (Tabela 1).

Com relação à procedência dos estudos publicados nos Anais do CBEn, o maior número de trabalhos publicados sobre a temática foram oriundos da região Sudeste (51\%), seguido de região Nordeste, com $22 \%$, região Sul (14\%) e região Centro-Oeste (5\%) (Gráfico 2). Ao analisar as publicações com relação à procedência de Estado da Federação, os resultados apontaram que a maioria dos trabalhos $(28 \%)$ foi realizada no estado do Rio de Janeiro, seguindo-se o estado de São Paulo (19\%), o estado do Ceará (9\%) e Rio Grande do Sul (8\%) (Gráfico 3). Com relação à afiliação dos autores dos trabalhos, os resultados indicaram que $16 \%$ dos autores pertenciam à Universidade Federal do Rio de Janeiro, seguindo-se a Universidade de São Paulo, que somou $10 \%$ do total das afiliações encontradas. Em 11\% dos resumos não estava disponível a identificação da instituição de origem do autor, e nos demais $63 \%$ dos trabalhos estavam indicadas outras afiliações que, agrupadas, não somaram $1 \%$ do total e por isso não são mencionadas.

Gráfico 2 - Distribuição dos resumos publicados nos anais do CBEn entre 1998 e 2008 de acordo com a região do país, São Paulo, 2010.

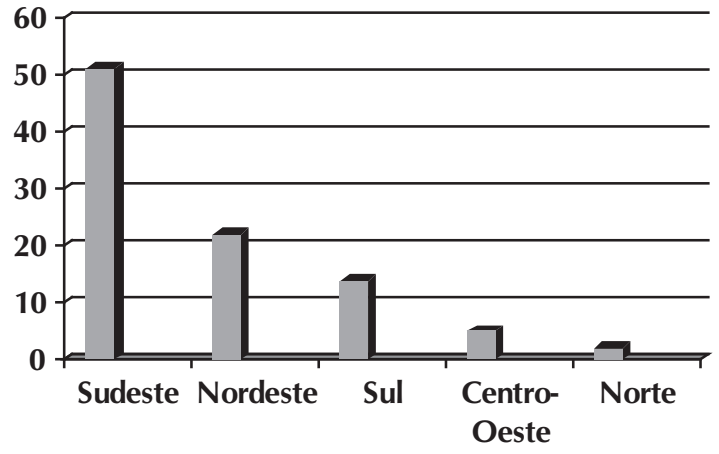

Gráfico 3 - Distribuição dos resumos publicados nos anais do CBEn entre 1998 e 2008 segundo o estado de procedência, São Paulo, 2010. (Outros)

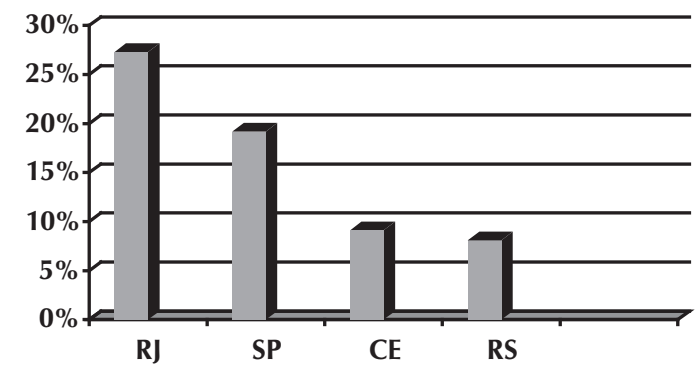

2) Categorias de análise segundo a exploração qualitativa dos resumos

Os resultados obtidos neste estudo permitiram agrupar os dados em sete categorias de análise, conforme descritas na sequência.

\section{Categoria I - Levantamentos populacionais}

$\mathrm{Na}$ categoria Levantamentos Populacionais, foram capturados 27 resumos publicados ( $27 \%$ do total); nos anais investigados, tais levantamentos realizaram-se principalmente em populações de usuários de serviços de saúde $(44 \%)^{(9)}$, estudantes de nível médio $(11 \%)^{(10)}$, universitários $(15 \%)^{(11)}$, populações específicas (12\%) - como motoristas ${ }^{(12)}$ e portadores de comorbidades crônicas ${ }^{(13)}$, e demandas espontâneas de homens, mulheres e jovens que responderam a instrumentos de triagem, como o CAGE (18\%). Além da prevalência do uso e abuso de álcool entre essas populações, os estudos apresentados abordaram, dentre outros aspectos, o padrão de consumo da substância pelas populações investigadas, repercussões sociais, econômicas e demográficas do uso e abuso do álcool.

\section{Categoria II - Assistência de Enfermagem}

$\mathrm{Na}$ categoria Assistência de Enfermagem, foram encontrados 32 resumos relacionados ao tema. Essa categoria abarca os trabalhos em que o foco principal é a assistência de enfermagem. Verificou-se que seus componentes se resumem a temas sobre assistência ao indivíduo na prevenção ao uso abusivo de álcool, educação em saúde, relacionamento enfermeiro/alcoolista, diagnóstico de enfermagem, assistência de enfermagem na Síndrome de abstinência, papel do enfermeiro no cuidado ao alcoolista ${ }^{(14)}$ e terapias alternativas direcionadas a essa população(15). Complementam a categoria os componentes que dizem respeito a assistência à família do indivíduo alcoolista, e ainda aparecem estudos relacionados a equipamentos da rede pública - esses últimos se ocuparam em estudar os modelos de atendimento e assistência ao usuário de álcool e outras drogas e a implantação de serviços de atenção a essa população, como os Centro de Atenção Psicossocial de Álcool e drogas - Caps Ad.

\section{Categoria III - Populações vulneráveis}

$\mathrm{Na}$ categoria populações vulneráveis, foram encontrados 27 resumos que se referiam a produções dos enfermeiros. Nessa categoria, pôde-se observar que as populações que despertaram maior interesse dos enfermeiros para estudo foram mulheres, adolescentes, universitários e populações específicas - motoristas de caminhão, taxistas e portadores de comorbidades clínicas, tais como diabetes e hipertensão.

Nos estudos relacionados à mulher, aparecem pesquisas principalmente sobre mulheres no ciclo gravídico, e esses estudos dizem respeito, especificamente, a filhos de gestantes que utilizaram o álcool durante a gravidez e conhecimentos destas sobre efeitos do álcool; compõe ainda essa categoria estudos realizados com adolescentes. Os aspectos mais estudados entre essas populações relacionam-se a investigação das causas e modos do início do uso do álcool ${ }^{(16)}$, fatores de risco, danos causados pelo álcool à saúde ${ }^{(17)} \mathrm{e}$, ainda, identificação do uso e estratégias de educação em saúde para esses indivíduos.

Entre os universitários, a atenção dos enfermeiros volta-se para o estudo dos fatores desencadeantes do consumo ${ }^{(13)}$, identificação de experiências prévias no consumo de álcool, opiniões e atitudes frente ao uso abusivo de álcool e outras drogas entre estudantes de enfermagem ${ }^{(18)}$, associação entre o 
uso do álcool e a direção de veículos, e prevalência de usuários de álcool na comunidade universitária ${ }^{(11)}$. Ainda no que se refere aos estudos com populações específicas, houve trabalhos relacionados a uso associado de álcool e anfetamina por motoristas de caminhão ${ }^{(12)}$, perfil do consumo de bebidas alcoólicas por deficientes visuais, incidência do uso de álcool entre técnicos de patologia clínica, e uso de bebidas alcoólicas entre taxistas.

\section{Categoria IV - Sentimentos, concepções e atitudes de en- fermeiros frente ao álcool e ao alcoolismo}

Na categoria Sentimentos, concepções e atitudes de enfermeiros frente ao atendimento ao alcoolista, encontraram-se seis resumos que mostraram que a maioria dos enfermeiros acredita que o alcoolismo é uma doença e que os usuários devem receber tratamento; que, porém, parte dos enfermeiros manifesta indiferença quanto a trabalhar com esses pacientes ${ }^{(19)}$; que as representações sociais de enfermeiros são oriundas de experiências práticas com essa clientela; e que, quanto às atitudes, os enfermeiros se mostraram moralistas e condenatórias com relação ao usuário. Os resultados da análise das produções vinculadas a essa categoria permitiram evidenciar que os enfermeiros concebem o alcoolismo como uma doença, entretanto preferem não trabalhar com esses pacientes.

\section{Categoria V - Ensino sobre álcool e outras drogas}

A categoria Ensino sobre Álcool e Outras Drogas constituiu-se de três resumos que trataram de investigar o conhecimento adquirido sobre a temática álcool e outras drogas no curso de graduação em enfermagem a partir da escala NEADA e mostrou que os estudantes de enfermagem apresentam atitude positiva sobre a assistência ao usuário; e que, porém, uma parte destes considera desagradável trabalhar com essa clientela. Outro estudo abordou o uso de um screening por estudantes de enfermagem para detecção do uso de álcool num hospital escola e observou a importância da utilização desses instrumentos no desenvolvimento de habilidades do aluno, desde a graduação, para reconhecer e detectar usuários de álcool, uma vez que este se trata de um problema de saúde pública e que esses alunos, como futuros profissionais de saúde, devem estar aptos, ao concluir o curso de graduação, a atender a essa demanda, a qual está inserida nos mais diversos serviços de saúde do País.

\section{Categoria VI - Concepções e vivências do alcoolista}

A categoria Concepções e Vivências do Alcoolista, que englobou os estudos que se referiram a percepção e vivência do alcoolista, se inseriu em sete resumos que relataram as necessidades psicossociais, de autoestima, liberdade, comunicação, lazer, aceitação e autoimagem como as mais afetadas no alcoolista e que levam à dependência, em representações sociais de alcoolistas abstêmios sobre os fatores que contribuíram para o uso abusivo do álcool ${ }^{(20)}$. A visão do alcoolista sobre si mesmo revela apenas sentimentos negativos como perdas e vulnerabilidades; os usuários relacionam os motivos de reinternação pela família aos sentimentos de fracasso, frustração e a recaídas ${ }^{(21)}$; no que se refere à compreensão do sentimento do alcoolista sobre o alcoolismo, verificou-se que os usuários são impotentes para lutar contra o consumo e que sua condição os leva a realizar projetos para melhorar sua qualidade de vida ${ }^{(22)}$; quanto ao conhecimento do alcoolista sobre a cirrose alcoólica, viu-se que os usuários sabiam da possibilidade de ela se desenvolver, mas pareciam ter pouca informação quanto aos danos irreversíveis causados pelo uso da bebida alcoólica e ao possível agravamento de seu quadro de saúde.

\section{Categoria VII - Alcoolismo e Família}

$\mathrm{Na}$ categoria Alcoolismo e Família foram encontrados seis resumos respectivos às repercussões do alcoolismo sobre os familiares de alcoolistas; nas questões compreender sentimentos e percepções diante das situações vivenciadas, as famílias se sentem desanimadas e desgastadas para enfrentar o problema ${ }^{(23)}$ e necessitam de maior apoio por parte das instituições para lidar melhor com seu familiar usuário; a relação família/alcoolista mostra que a maioria das famílias apresenta disponibilidade para cuidar de seus parentes, embora manifeste intensa ansiedade e estresse; a importância de redes de suporte social utilizadas por famílias desses indivíduos, o desuso desse recurso no PSF e o perfil dos familiares de alcoolistas apontam que o uso do álcool pode ser desencadeado e interferir no lar a partir da perda de um líder familiar, levando à desorganização desta estrutura(24).

\section{DISCUSSÃO}

A análise realizada a partir dos resultados encontrados no levantamento das produções de enfermeiros sobre o tema álcool e alcoolismo na década de 1998 a 2008 possibilitou constatar que pouca atenção tem sido dispensada à questão pelos profissionais enfermeiros, o que corrobora os apontamentos de outros autores ${ }^{(6,25)}$ sobre a escassez de publicações dos enfermeiros brasileiros a respeito do assunto.

É possível que as questões relacionadas ao álcool e alcoolismo despertem pouco interesse dos enfermeiros para a realização de estudos ou que, quando são realizados, estes não resultem em interesse suficiente dos autores para divulgação e publicações. Com relação a essas hipóteses, estudos evidenciam $^{(6)}$ que, devido ao caráter multidisciplinar do assunto, os pesquisadores da enfermagem podem estar priorizando outras áreas do conhecimento para realização de suas pesquisas e deixando as questões relacionadas ao álcool e ao alcoolismo em segundo plano. Frente a essa constatação, pesquisadores ${ }^{(26)}$ sugeriram que as universidades enfatizem o tema na graduação e no pós-graduação, pois, além de atualizar o acervo bibliográfico sobre a temática, essa estratégia poderia incentivar o desenvolvimento de projetos de extensão e pesquisa sobre alcoolismo que realmente representem impacto social.

Apesar da escassez em comparação com publicações de outras áreas - o que pode ser considerado uma limitação desse estudo -, a análise dos dados permitiu verificar um sutil aumento dos trabalhos sobre álcool e alcoolismo apresentados no Congresso Brasileiro de Enfermagem na última década. Diante disso, vale ressaltar que a produção científica da enfermagem, a partir da década de 1990, apresentou um grande avanço em 
geral, visto que neste espaço de tempo os critérios de avaliação dos programas de pós-graduação, estipulados pela CAPES, tornaram-se mais rigorosos ao exigir mais publicações científicas e diminuição no tempo de obtenção das titulações de Mestres e Doutores. Além disso, surgiram novos programas de pós-graduação $^{(6)}$ e, consequentemente, a ampliação de linhas de pesquisas na área de álcool e outras drogas. No que se refere à procedência desses estudos, a maioria (51\%) foi realizada na região Sudeste, e o estado do Rio de Janeiro obteve destaque, seguido do estado de São Paulo. Esses dados são consistentes com aqueles encontrados em estudo semelhante ${ }^{(6)}$. A explicação para esse fenômeno pode estar relacionada ao fato de a maior concentração de pesquisadores e grupos de pesquisas dessa área estar alocada nesses estados.

A análise dos resultados evidenciou que os enfermeiros tiveram maior interesse por temas referentes a assistência de enfermagem, mais especificamente assistência de enfermagem ao indivíduo. Resultado semelhante foi encontrado em levantamento realizado nos Anais de um evento de enfermagem em saúde mental ${ }^{(27)}$, que constatou que as práticas assistenciais têm constituído os temas mais abordados pelos participantes, seguido de pesquisa e ensino. Este resultado poderia ser explicado pelo fato de refletir as experiências do profissional na atenção ao indivíduo com problemas relacionados ao álcool ${ }^{(24)}$. Esses resultados são consistentes também com outros observados em um estudo afim ${ }^{(6)}$, que evidenciou que as temáticas relativas às aqui identificadas constituíam a tendência da produção de enfermagem na área do álcool e outras drogas. Além de apontar carências de pesquisas que envolvam idosos, bem como temas que abordam o álcool e a violência, este estudo reforça as evidências sobre as tendências da pesquisa da enfermagem nessa área.

Investigando as questões abordadas pelos enfermeiros divulgadas nos resumos do CBEn, pode-se observar ainda que, entre a maioria das produções que envolvem populações vulneráveis, se destacaram os trabalhos sobre universitários e que também aparecem com frequência estudos a respeito de mulheres e adolescentes. Estes dados são consistentes com outro levantamento ${ }^{(6)}$, que evidenciou que os pesquisadores enfermeiros, quando estudam grupos populacionais, enfatizam os jovens de maneira geral. Segundo as autoras ${ }^{(6)}$, isto se deve ao fato de o período de transição para a universidade ser apontado como uma fase de vulnerabilidade, o que propicia o incremento ao uso de álcool e outras drogas ${ }^{(28)}$.

As investigações que envolvem mulheres foram frequentes nesse levantamento e perfizeram quase um terço das investigações realizadas. No entanto, conforme apontou trabalho anterior $^{(6)}$, parte significativa desses estudos se deu com populações de gestantes. Ao contrário do que foi encontrado naquele estudo, observou-se que, embora em número reduzido, alguns tratados envolveram populações de usuárias de álcool, o que evidencia que essas pesquisas têm sido realizadas pelos enfermeiros, mas não se revertem, no entanto, em publicações de veículos de grande circulação, portanto, não indexadas.

Quanto às produções que abordaram sentimentos, concepções e atitudes de enfermeiros sobre o uso e abuso de álcool, a preocupação desses profissionais em investigar esta temática pode estar relacionada à sua sensibilização para com a questão, pois cada vez mais eles têm tido contato com essa realidade, apesar de muitos ainda se encontrarem despreparados para o enfrentamento da situação, o que tende a lhes provocar sentimentos e atitudes, fato que despertou o interesse dos pesquisadores na última década(4), que pode ser evidenciado com o aumento de resumos publicados nos Anais do CBEn sobre essa questão, a partir do ano de 2001.

Na categoria Ensino sobre álcool e outras drogas, foram poucos os resumos que abordaram esse ensino na enfermagem. Embora o uso de álcool constitua o principal risco de saúde nos países em desenvolvimento e, na região das Américas, ele se configure como dos principais fatores de risco entre os 27 avaliados na carga de morbidade ${ }^{(29)}$, parece que esse fato não tem repercutido na formação dos futuros enfermeiros, já que a produção sobre a temática é escassa, o que chama a atenção, dada a importância do preparo do profissional de saúde, dentre eles, o enfermeiro, no desenvolvimento de habilidades na assistência a essa clientela. Assim, isto é considerado um reflexo da atual conjuntura do ensino da temática álcool e outras drogas nos cursos de graduação em enfermagem, apontado pelos pesquisadores da área( ${ }^{(30)}$ como insuficiente, o que, em última análise, repercute na carência de estudos que envolvem o tema 'ensino sobre dependências' em artigos apresentados e publicados nos Anais do CBEn.

Apesar das limitações, representadas pela investigação em fontes de um único evento, o tamanho da amostra envolvida e a escassez de trabalhos publicados no CBEn referentes ao álcool e outras drogas, em comparação com as publicações de outras áreas, este estudo traz avanço para o conhecimento sobre a produção da enfermagem sobre a temática álcool e alcoolismo, pois abrange fontes ainda não investigadas pela enfermagem e fornece dados que contribuem para o mapeamento da produção da área nesta temática. Além disso, apresenta o compilamento da produção sobre o tema no maior evento científico da enfermagem brasileira no período de uma década (1998-2008), e seus resultados serviram também de subsídio para a realização de estudos da mesma natureza que estão em andamento e que buscam monitorar como anda a produção sobre o alcoolismo e as questões associadas no Congresso Brasileiro de Enfermagem a cada cinco anos. Os resultados observados no último quinquênio estão em análise e brevemente serão publicados. Frente a esses apontamentos, os dados apurados que apresentamos se tornam importantes, sobretudo quando se considera a escassez de estudos que tenham se ocupado de levantar e caracterizar tal produção, bem como por possibilitar o monitoramento da produção sobre a temática no maior evento da enfermagem brasileira.

\section{CONCLUSÕES}

Apesar de pouco expressiva, a produção de enfermeiros sobre o álcool e alcoolismo apresentada no CBEn mostrou um aumento considerável nos últimos cinco anos, sobretudo no que diz respeito às questões relacionadas à assistência a esse tipo de clientela. Os Anais do CBEn constituem fontes importantes de dados de pesquisas realizadas por enfermeiros 
na temática álcool e outras drogas, mas nem sempre os trabaIhos apresentados revertem em publicações indexadas, o que torna difícil o acesso aos pesquisadores da área. Sugere-se que outros estudos desta natureza sejam realizados, na busca de se identificar como têm ocorrido a produção e a divulgação das pesquisas na enfermagem sobre o álcool e o alcoolismo, uma vez que a compilação desses estudos pode fornecer subsídios à construção de um corpus de conhecimento na área.

\section{REFERÊNCIAS}

1. Organização Mundial Sáude. Relatório mundial da saúde. Saúde mental: nova concepção, nova esperança [monografia na internet]. Geneva: WHO; 2002 [acesso em 15 nov 2008]. Disponível em: www.who.int/whr/2001/ en/whr01_djmessage_po.pdf .

2. Organización Panamericana de La Salud. Desenvolvimento Sustentável e Saúde Ambiental [monografia na Internet]. OPAS; 2007. [acesso em 27 jan 2010] Disponível em: http://www.paho.org/hia/homepor.html.

3. Carlini EA, Galduróz JCF, Noto AR, Nappo SA. II Levantamento domiciliar sobre uso de drogas no Brasil - 2005. Centro Brasileiro de Informações Sobre Drogas Psicotrópicas; 2006.

4. Lucca DM, Vargas D, Vargas D. As concepções de enfermeiros de hospital geral frente as questões relacionadas ao álcool e ao alcoolismo. Rev Enferm Bras 2006;5(4):260-7.

5. Ministério da Saúde. Secretaria Nacional Antidrogas. Glossário de álcool e drogas. Brasília; DF: O Ministério; 2006.

6. Luis MAV, Lunetta ACF. Álcool e outras drogas: Levantamento preliminar sobre a pesquisa produzida no Brasil pela enfermagem. Rev Latino-Am Enferm 2005;13(n. oesp.):1229-30.

7. Mancia JR, Padilha MICS, Ramos FRS, Cordova FP, Amaral NV. Congresso Brasileiro de Enfermagem: sessenta anos de historia. Rev Bras Enferm 2009;62(3):471-9.

8. Minayo MCS. Pesquisa Social: teoria, método e criatividade. 23. ed. Petrópolis: Vozes; 1994.

9. Rosa AJS, Caetano FB, Herculian JG, Oda MT. Mitos e verdades sobre o alcoolismo - a opinião dos usuários em um serviço de saúde. In: Anais do $53^{\circ}$ Congresso Brasileiro de Enfermagem; 2001 out 9-14; Curitiba, Brasil. Curitiba: Associação Brasileira de Enfermagem; 2001.

10. Silva BS, et al. Consumo de bebidas alcoólicas por adolescentes: subsídios para a atuação do enfermeiro na escola. In: Anais do $60^{\circ}$ Congresso Brasileiro de Enfermagem; 2008 nov 3-6; Belo Horizonte, Brasil. Belo Horizonte: Associação Brasileira de Enfermagem; 2008.

11. Pertussatti CA, Souza CMS, Pazotti GM, Attilio JS, Reis CB. Utilização de bebidas alcoólicas entre acadêmicos do $1^{\circ}$ ano dos cursos de graduação da universidade estadual de Mato Grosso do Sul, unidade universitária de Dourados/MS. In: Anais do $58^{\circ}$ Congresso Brasileiro de Enfermagem; 2006 nov 5-9; Salvador, Brasil. Salvador: Associação Brasileira de Enfermagem; 2006.

12. Fonseca AD. Anfetamina associada a álcool: efeito morte. In: Anais do $53^{\circ}$ Congresso Brasileiro de Enfermagem; 2001 out 9-14; Curitiba, Brasil. Curitiba: Associação Brasileira de Enfermagem; 2001.
13. Assis GO. O consumo do álcool e as doenças cardiovasculares - uma analise sob o olhar da enfermagem. In: Anais do $59^{\circ}$ Congresso Brasileiro de Enfermagem; 2007; Brasília, Brasil. Brasília: Associação Brasileira de Enfermagem; 2007.

14. Perotta SM. A inserção da enfermagem em Caps de álcool e outras drogas. In: Anais do $58^{\circ}$ Congresso Brasileiro de Enfermagem; 2006 nov 5-9; Salvador, Brasil. Salvador: Associação Brasileira de Enfermagem; 2006.

15. Ramos ASMB, Nascimento AM, Cutrim AP, Pinheiro JFS, Viveiros MTM. Grupo de apoio provida: Uma possibilidade de reabilitação do usuário de álcool e outras drogas. In: Anais do $58^{\circ}$ Congresso Brasileiro de Enfermagem; 2006 nov 5-9; Salvador, Brasil Salvador: Associação Brasileira de Enfermagem; 2006.

16. Cardoso AMV, Francheschini M. Adolescencia, álcool e escola. In: Anais do $50^{\circ}$ Congresso Brasileiro de Enfermagem; 1998; Salvador, Brasil. Salvador: Associação Brasileira de Enfermagem; 1998.

17. Gonçalves $M$, et al. Os malefícios causados pelo uso de álcool e do fumo. In: Anais do $55^{\circ}$ Congresso Brasileiro de Enfermagem; 2003 nov 10; Rio de Janeiro, Brasil. Rio de Janeiro: Associação Brasileira de Enfermagem; 2003.

18. Carvalho MTC, Oliveira ISC. Uso de álcool e outras drogas entre as estudantes de enfermagem. In: Anais do $55^{\circ}$ Congresso Brasileiro de Enfermagem; 2003; Rio de Janeiro, Brasil. Rio de Janeiro: Associação Brasileira de Enfermagem; 2003.

19. Lucca DM, Vargas D. Concepções de enfermeiros frente ao paciente alcoolista e o alcoolismo. In: Anais do $57^{\circ}$ Congresso Brasileiro de Enfermagem; 2005 nov 3-7; Goiânia, Brasil. Goiânia: Associação Brasileira de Enfermagem; 2005.

20. Souza MJ, Silva SED. Alcoolismo: Representações sociais de alcoolistas abstêmios. In: Anais do $54^{\circ}$ Congresso Brasileiro de Enfermagem; 2002; Fortaleza, Brasil. Fortaleza: Associação Brasileira de Enfermagem; 2002.

21. Reis ASS, Tortori D, Melo RM, Regazzi I. As reinternações como instrumento de avaliação do tratamento pautado na política de redução de danos: Uma visão do alcoolista. In: Anais do $55^{\circ}$ Congresso Brasileiro de Enfermagem; 2003 nov 10; Rio de Janeiro, Brasil. Rio de Janeiro: Associação Brasileira de Enfermagem; 2003.

22. Costa MS, Resende SEM, Silva MSP. O sentimento das pessoas que vivenciam a dependência química do alcool. In: Anais do $57^{\circ}$ Congresso Brasileiro de Enfermagem; 2005 nov 3-7; Goiânia, Brasil. Goiânia: Associação Brasileira de Enfermagem; 2005.

23. Rodrigues CO, Antonio PS. O alcoolismo e suas repercussões sobre a família. In: Anais do $58^{\circ}$ Congresso 
Brasileiro de Enfermagem; 2006 nov 5-9; Salvador, Brasil. Salvador: Associação Brasileira de Enfermagem; 2006.

24. Silva Júnior IA, Freire FFS, Oliveira AAL, Sousa JM, Lima LHO. Dependência química: o álcool na influência do ambiente familiar. In: Anais do $58^{\circ}$ Congresso Brasileiro de Enfermagem; 2006 nov 5-9; Salvador, Brasil Salvador: Associação Brasileira de Enfermagem; 2006.

25. Oliveira LCG. Levantamento da produção de enfermeiros sobre álcool e alcoolismo nos anais de resumos do congresso brasileiro de enfermagem, no período de 1995 a 2005. Ribeirão Preto. Monografia [Especialização] - Escola de Enfermagem de Ribeirão Preto da USP; 2006.

26. Donato M, Zeitoune RCG. Reinserção do trabalhador alcoolista: percepção, limites e possibilidades de intervenção do enfermeiro no trabalho. Esc Anna Nery Rev Enferm 2006;10(3):399-407.

27. Munari DB, Oliveira NF, Saeki T, Souza MCBM. Análise da produção científica dos encontros de pesquisadores em enfermagem psiquiátrica e saúde mental. Rev Latino-Am Enferm 2008:16(3):471-6.

28. Peuker AC, Fogaça J, Bizarro L. Expectativas e beber problemático entre universitários. Psicol Teori Pesqui 2006;22(2):193-200.

29. Organización Panamericana de La Salud. Salud en las Américas 2007: Volumen I - Regional. Washington; 2007.

30. Lopes GT, Luis MAV. A formação do enfermeiro e o fenômeno das drogas no estado do Rio de Janeiro - Brasil: atitudes e crenças. Rev Latino-Am Enferm 2005;13(n. ${ }^{\circ}$ esp.). 